\section{Serial brain magnetic resonance imaging in a patient with invasive streptococcal infection with ventriculitis and choroid plexitis}

\author{
Jaewoon Chung, MD' ; Ji Young Lee, MD, PhD²; Young Seo Kim, MD, PhD' \\ 'Department of Neurology, Hanyang University College of Medicine, Seoul, Korea \\ ${ }^{2}$ Department of Radiology, Hanyang University College of Medicine, Seoul, Korea
}

\section{Journal of Neurocritical \\ Care \\ IMAGES IN \\ NEUROCRITICAL CARE \\ Received: October 11, 2021 \\ Revised: November 26, 2021 \\ Accepted: November 26, 2021 \\ Corresponding Author: \\ Young Seo Kim, MD, PhD \\ Department of Neurology, Hanyang \\ University College of Medicine, 222-1 \\ Wangsimni-ro, Seongdong-gu, Seoul \\ 04763, Korea \\ Tel: +82-2-2290-8375 \\ Fax: +82-2-2299-2391 \\ E-mail: aescula@hanmail.net}

A 39-year-old previously healthy man visited the hospital with fever, myalgia, and vomiting. His systolic blood pressure was below $60 \mathrm{mmHg}$, and he was admitted to the intensive care unit with the suspicion of septic shock. After admission, Streptococcus pneumonia was found in his blood, and treatment with vancomy$\operatorname{cin} 2 \mathrm{~g} /$ day and ceftriaxone $4 \mathrm{~g} /$ day was initiated. Following antibiotic treatment for 4 days, his mentation deteriorated to a stupor. Brain magnetic resonance imaging (MRI) indicated hydrocephalus, ventriculitis, and choroid plexitis (Fig. 1). Cerebrospinal fluid (CSF) examination showed normal opening pressure (180 $\mathrm{mmHg}$ ), pleocytosis (white blood cell count, 280; neutrophils, $55 \%)$, high protein level (1,448 mg/dL), and low glucose levels (CSF, $54 \mathrm{mg} / \mathrm{dL}$; serum, $135 \mathrm{mg} / \mathrm{dL}$ ). Consequently, a high dose of steroids (dexamethasone, $40 \mathrm{mg} /$ day) was prescribed together with antibiotics. After 14 days of antibiotic treatment, his general condition improved.

Brain MRI findings depicting pyogenic ventriculitis typically include ependymal thickening and enhancement with T2 prolongation surrounding the ventricle, hydrocephalus, and debris within the dependent aspect of the ventricles $[1,2]$. In addition, diffusion restriction and swelling of the choroid plexus are suggestive of choroid plexitis [2,3]. Although the MRI findings of our patient seemed more critical than previous reports, the patient experienced a good outcome with antibiotics [4].

\section{ARTICLE INFORMATION}

\section{Ethics statement}

This case was approved by the Institutional Review Board of the Hanyang University Hospital (IRB No. HYUH 2021-10-010). Informed consent from a patient was waived by the IRB.

\section{Conflict of interest}

No potential conflict of interest relevant to this article.

\section{ORCID}

Jaewoon Chung https://orcid.org/0000-0001-6936-9023

Ji Young Lee https://orcid.org/0000-0003-1181-8070

Young Seo Kim https://orcid.org/0000-0002-7050-3426

\section{Author contributions}

Conceptualization: YSK. Data curation: JC, JYL. Formal analysis: 


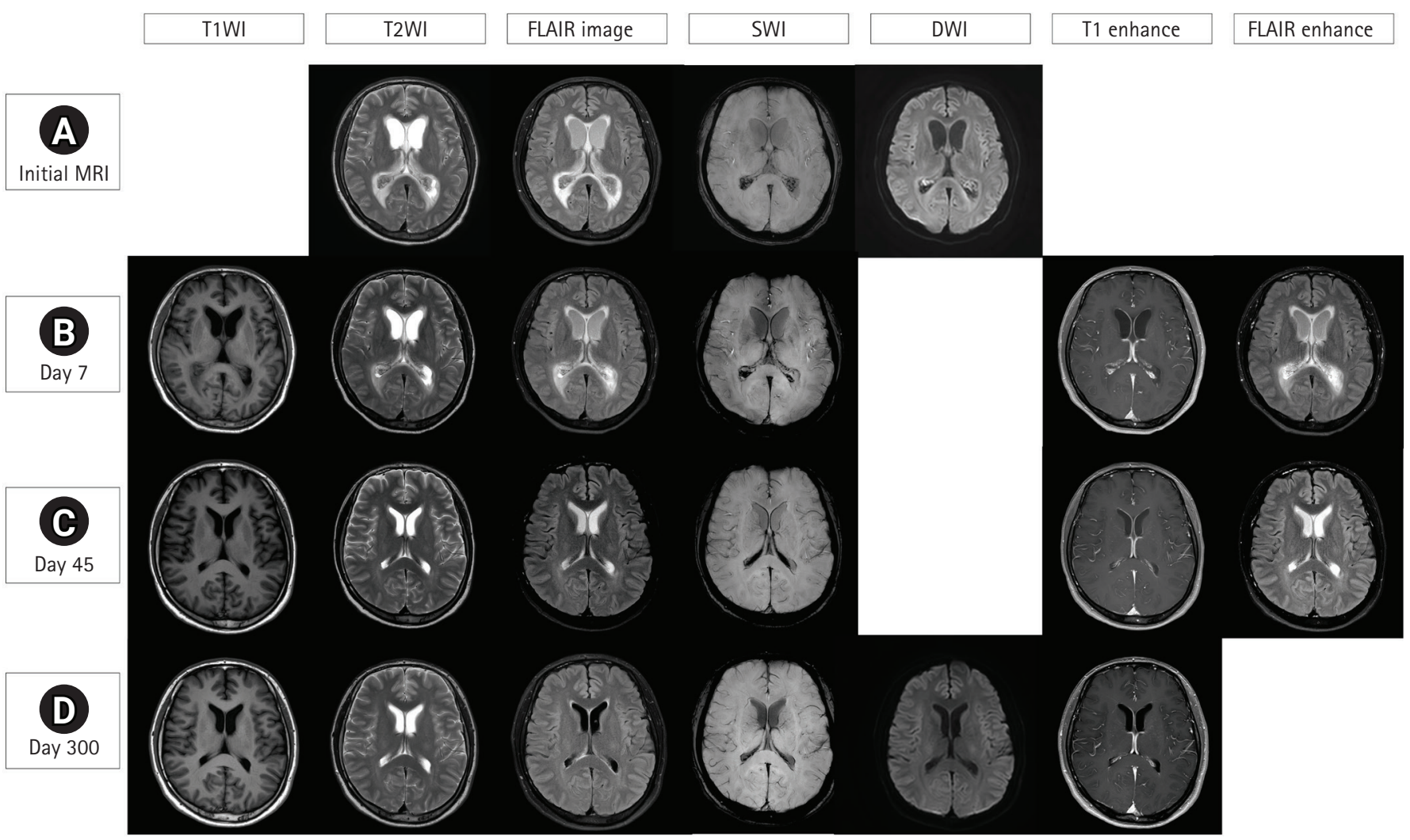

Fig. 1. Serial brain magnetic resonance imaging (MRI) findings of invasive streptococcal sepsis with ventriculitis and choroid plexitis. Initial brain images reveal hydrocephalus with severe interstitial edema and non-suppressed high-signal fluid in the lateral ventricle on fluidattenuated inversion recovery (FLAIR) imaging, suggesting pyogenic ventriculitis. In addition, restricted diffusion on diffusion-weighted imaging (DWI) and low signal intensity on susceptibility-weighted imaging (SWI) in both choroid plexuses indicate choroid plexitis with hemorrhaging (A). Although the patient's mental status improved after administration of intravenous vancomycin and ceftriaxone, no significant change is observed in the brain MRI on day 7 after admission (B). The brain MRI of day 45 shows improved hydrocephalus, interstitial edema, ventriculitis, and choroid plexitis; however, a high signal of cerebrospinal fluid on FLAIR imaging is noted (C). Finally, on day 300 , a normal brain MRI is observed (D).

YSK. Methodology: YSK. Project administration: YSK, JYL. Visualization: JYL. Writing-original draft: JC. Writing-review \& editing: YSK.

\section{REFERENCES}

1. Mohan S, Jain KK, Arabi M, Shah GV. Imaging of meningitis and ventriculitis. Neuroimaging Clin N Am 2012;22:557-83.

2. Hazany S, Go JL, Law M. Magnetic resonance imaging of infec- tious meningitis and ventriculitis in adults. Top Magn Reson Imaging 2014;23:315-25.

3. Pezzullo JA, Tung GA, Mudigonda S, Rogg JM. Diffusion-weighted MR imaging of pyogenic ventriculitis. AJR Am J Roentgenol 2003;180:71-5.

4. Woehrl B, Linn J, Lummel N, Pfefferkorn T, Koedel U, Pfister HW, et al. Pneumococcal meningitis-associated pyogenic ventriculitis. J Infect 2015;70:311-4. 